\title{
Prediction Model of Pathologic Central Lymph Node Negativity in cNO Papillary Thyroid Carcinoma
}

\author{
Xiujie Shu ${ }^{1}$, Lingfeng Tang ${ }^{1}$, Daixing $\mathrm{Hu}^{2}$, Yuanyuan Wang ${ }^{1}$, Ping $\mathrm{Yu}^{1}$, Zhixin Yang ${ }^{1}$, \\ Chang Deng ${ }^{1}$, Denghui Wang ${ }^{1}$ and Xinliang Su ${ }^{1 *}$ \\ ${ }^{1}$ Department of Endocrinology and Breast Surgery, The First Affiliated Hospital of Chongqing Medical University, \\ Chongqing, China, ${ }^{2}$ Department of Breast and Thyroid Surgery, The Second Affiliated Hospital of Chongqing Medical \\ University, Chongqing, China
}

Background: Most patients with papillary thyroid carcinoma (PTC) have an excellent prognosis. Although central lymph node invasion is frequent, management via central lymph node dissection (CLND) remains controversial. The present study retrospectively investigated independent predictors of pathologic central lymph node negativity (pCLN-) and established a prediction model for pCLN- in clinical lymph node negativity (cNO) PTC.

Methods: A total of 2,687 patients underwent thyroid surgery for cNO PTC from 2013 to 2018 at the First Affiliated Hospital of Chongqing Medical University, and lobectomy plus ipsilateral CLND was the basic surgical extent. Clinicopathological characteristics were reviewed and analyzed. Univariate and multivariate analyses were performed to identify factors related to pCLN-. A prediction model was established based on the results of multivariate analyses.

Results: The $\mathrm{PCLN}$ - rate was $51.5 \%(1,383 / 2,687)$. Multivariate analysis revealed that sex, age, thyroid stimulating hormone (TSH), size, location, laterality, unifocality and extrathyroidal extension negativity (ETE-) were independent predictors of pCLN-. The nomogram showed good discriminative ability ( $\mathrm{C}$-index: 0.784 and 0.787 in derivation and validation groups, respectively) and was well calibrated. We quantified the clinical usefulness of the nomogram by decision curve analysis. The median length of follow-up was 30 (range 12-83) months, and 190 cases were lost, with a follow-up rate of $92.9 \%$ $(2,497 / 2,687)$. Of the 2,687 patients included, 21 (0.8\%) experienced recurrence.

Conclusion: This nomogram, which integrates available preoperative clinicopathological features and intraoperative frozen biopsy outcomes, is a reliable tool with high accuracy to predict PCLN- in cNO PTC.

Keywords: papillary thyroid carcinoma, nomogram, pathologic lymph node negativity, prognosis, central lymph node 


\section{INTRODUCTION}

Thyroid cancer is the most common endocrine malignancy, and papillary thyroid carcinoma (PTC) accounts for more than $90 \%$ of cases. The development of ultrasound and fine needle aspiration cytology rapidly improved the diagnosis of PTC in recent years (1-3). Most of PTCs are indolent and carry an excellent prognosis with low mortality. However, early-stage metastasis in cervical lymph nodes may occur, especially in the central compartment (Level VI), which is bound superiorly by the hyoid bone, inferiorly by the innominate artery, and laterally by the common carotid artery. Overall, accurate evaluation of lymph node status is of great significance for PTC treatment, TNM staging and risk stratification, but current methods for evaluating central lymph node (CLN) status have several limitations. Inconsistencies between clinical and pathologic evaluations may also exist. According to the 8th edition of the American Joint Committee on Cancer (AJCC) TNM staging, N0 is subdivided into NOa and NOb. N0a, also known as pN0, refers to negative lymph nodes confirmed by cytology or histology, and N0b, also known as cNO, indicates no metastatic lymph nodes (normal lymph nodes or nonspecific lymphadenitis) found on clinical and imaging examinations (4-7). Ultrasound is the most commonly used tool for the clinical evaluation of lymph nodes. The ultrasonic signs of typical lymph node metastasis primarily include a round shape, disappearance of the lymphatic hilum, mass hyperechoic regions, calcification foci (primarily microcalcification; coarse calcification is rare), cystic changes, and peripheral or mixed blood flow distribution signals. None of these features alone is sufficient to diagnose all metastatic lymph nodes, and the accuracy of the evaluation is limited based on considering the experience of clinicians, the function of instruments and heterogeneity among patients, the accuracy of the evaluation is limited (8-10). Therefore, only some patients with cN0 PTC have occult central lymph node metastasis (CLNM). The 2015 American Thyroid Association (ATA) and 2021 National Comprehensive Cancer Network (NCCN) guidelines recommend that $\mathrm{T} 1$ and $\mathrm{T} 2 \mathrm{cN} 0$ PTC should not be treated using prophylactic central lymph node dissection (CLND). However, surgical management of cN0 PTC remains controversial because of the presence of occult metastatic lymph nodes in cNO PTC and the debate surrounding the effect of these lymph nodes on recurrence and prognosis $(5,11-14)$. Balancing the risks and benefits of lymph node dissection is crucial for individualized and precise treatment. For cNO PTC with benign CLNs, it is reasonable to avoid prophylactic CLND, which protects the cervical tissue, reduces the extent of surgery and the corresponding complications, and facilitates the recovery

Abbreviations: $\mathrm{cN} 0$, clinical lymph node negativity; pN0, pathologic lymph node negativity; pCLN-, pathologic central lymph node negativity; PTC, papillary thyroid carcinoma; PTMC, papillary thyroid microcarcinoma; CLN, central lymph node; CLNM, central lymph node metastasis; CLND, central lymph node dissection; SET, scarless endoscopic thyroidectomy; BMI, body mass index; TSH, thyroid stimulating hormone; Tg, thyroglobulin; HT, Hashimoto's thyroiditis; ETE-, extrathyroidal extension negativity (tumor confined to the thyroid); IQR, interquartile range; CLNY,central lymph node yield; CLNR, central lymph node ratio; AUC, area under curve; DFS, disease-free survival; OR, odd ratio; CI, confidence index. of patients after surgery. This approach essentially promotes the development of scarless endoscopic thyroidectomy (SET). However, there have been few studies on the clinicopathological characteristics of these patients and a practical quantitative tool is urgently needed to identify these patients. We aim to establish a prediction model of pathologic central lymph node negativity (pCLN-) in patients with cNO PTC.

\section{MATERIALS AND METHODS}

\section{Patient Collection}

The local institutional ethics committee approved this retrospective study. Because there was no patient interest or privacy, informed consent was waived after careful review by the ethics committee. A total of 3,916 patients underwent surgery for thyroid diseases at the Department of Endocrine and Breast Surgery, The First Affiliated Hospital of Chongqing Medical University, between January 2013 and December 2018. All patients underwent at least a physical examination and cervical ultrasound examination before surgery. We used the $\mathrm{cN} 0$ assessment criteria of Kouvaraki et al. (1). Physical examination did not address enlarged lymph nodes or swollen lymph nodes that had a soft texture (2). Ultrasound examination showed no enlarged lymph nodes or swollen lymph nodes were oval and flat, regularly shaped, with clear boundaries between the cortex and medulla or a clear central fat hilum and no obvious malignant signs (15). Patients with any of the following conditions were excluded: incomplete clinicopathological data (403), CN1 confirmed by preoperative examination (536), non-PTC histology (34), reoperation or other head and neck surgery history (105), drug therapy for hyperthyroidism or hypothyroidism (27), absence of CLND (79), distant metastasis (14), adolescent (22), radiation exposure or family history of PTC (9). A total of 2,687 eligible patients were ultimately included in this study (Figure 1).

\section{Surgical Procedure}

For all patients enrolled, lobectomy plus ipsilateral CLND was the basic surgical treatment. Total thyroidectomy plus bilateral CLND was further performed for cases with bilateral tumors, tumors with a maximum diameter $>2 \mathrm{~cm}$ or intraoperative frozen section biopsy revealing CLNM. We routinely performed CLN intraoperative frozen section biopsy. Three pathologists blindly and independently diagnosed all resected thyroid and lymph node specimens.

\section{Demographic and Clinicopathological Data}

Data for sex, age, body mass index (BMI), thyroid stimulating hormone (TSH), size, location, unifocality, laterality, Hashimoto's thyroiditis (HT) and extrathyroidal extension negativity (ETE-) were collected and analyzed. The diameter of the largest lesion was recorded as the size, and the central location of the largest lesion was recorded as the location. When the tumor occupied almost the entire lobe, its location was recorded as "whole". Notably, ipsilateral lesions indicated the presence of laterality, and the presence of only one lesion in one lobe indicated unifocality. Tumors in which growth was confined to the thyroid gland without capsule invasion 


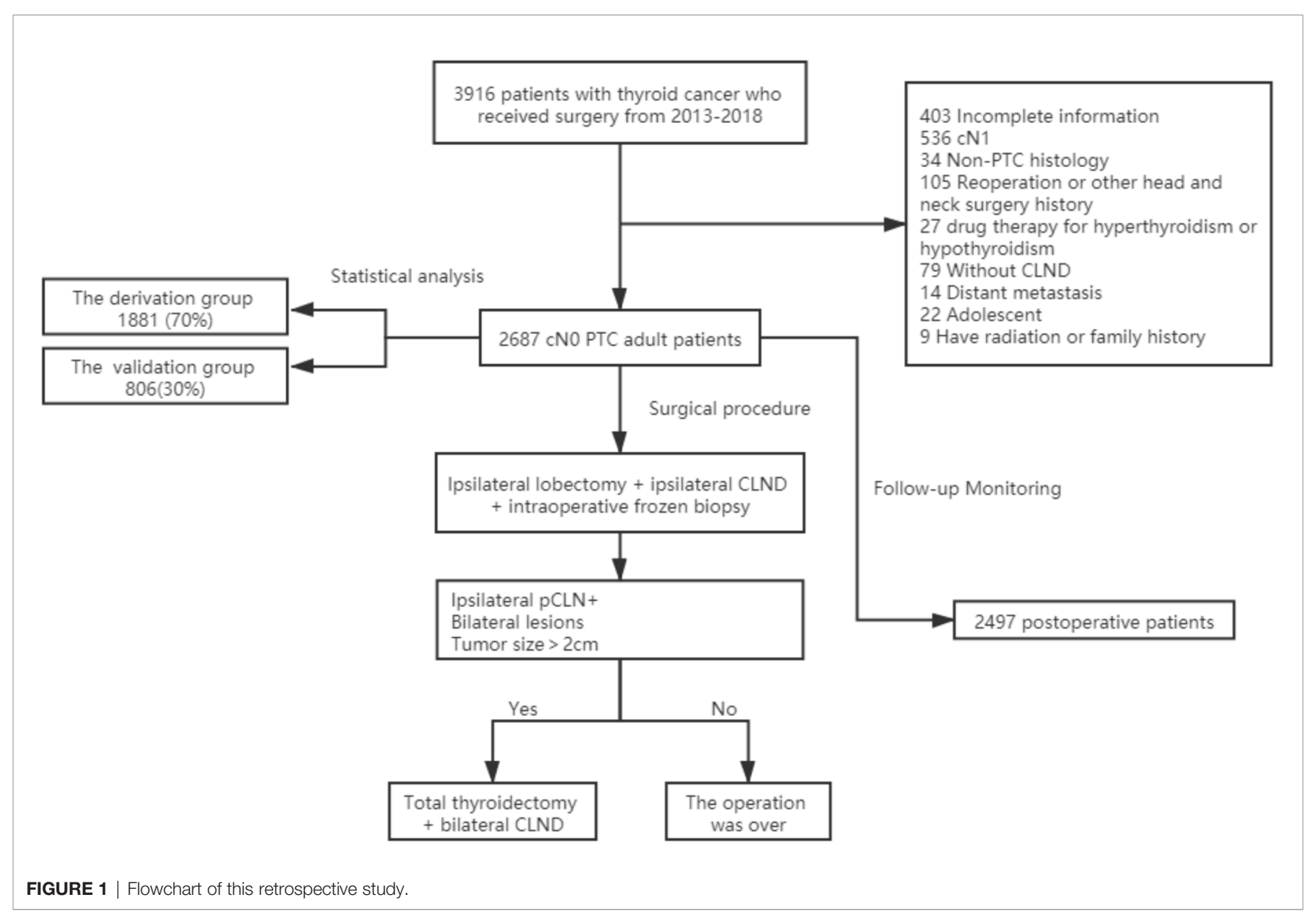

were defined as ETE-, minimal ETE confirmed by intraoperative frozen section biopsy was not considered ETE-.

\section{Follow-up Monitoring}

For all patients, the first follow-up was 1 month after surgery, and evaluations included a physical examination and measurement of serum TSH levels. Measurements of serum thyroglobulin (Tg) and Tg antibody levels were only performed for patients who underwent total thyroidectomy. The second follow-up was 6 months postoperatively. Cervical ultrasound examination was also performed for the first time. Regular evaluations were performed every 6-12 months thereafter. Patients with clinical evidence of recurrence, an unstimulated $\mathrm{Tg}$ level $\geq 2 \mathrm{ng} / \mathrm{mL}$, a stimulated $\mathrm{Tg}$ level $\geq 20 \mathrm{ng} / \mathrm{mL}$ or serial increases in serum $\mathrm{Tg}$ (with negative Tg antibody), or an imaging abnormality were suspected of recurrence $(16,17)$. We performed fine needle aspiration in these patients and reoperations to identify the pathology of the recurrence.

\section{Statistical Analysis}

The Mann-Whitney test was performed to compare differences in nonnormally distributed continuous variables (e.g., age, BMI, TSH, and size) between the two groups. Continuous variables with significant differences were converted into categorical variables. The chi-square test or Fisher's exact test was used to compare categorical variables. The Excel random function was used to assign values to each patient, and the patients corresponding to these values were randomly sorted. Therefore, a total of 2,687 patients were randomly divided into a derivation group (70\%) and a validation group (30\%). Multivariate analyses were performed using logistic regression analysis to estimate the statistical significance of the relationships between pCLN- and the clinicopathological characteristics in the derivation group. $\mathrm{P}<0.05$ was considered a statistically significant difference. A nomogram was developed based on the results of these analyses, and the predictive ability of the nomogram was measured using the receiver operating characteristic (ROC) curve, concordance index (C-index) and calibration plot. To evaluate the clinical usefulness of the nomogram, decision curve analysis (DCA) was performed to calculate the net benefits at different threshold probabilities. The Kaplan-Meier method was used to estimate disease-free survival (DFS) rates. All statistical analyses were performed using SPSS version 25.0 (IBM Corp., USA), and graphs were generated using $\mathrm{R}$ software.

\section{RESULTS}

\section{Patient Characteristics}

A total of 2,687 patients were included in the analysis (684 males and 2,003 females). The median age at diagnosis was 43 years 
(range 18-85 years). The median BMI level was 23.07 (range 15.56-36.98), and the mean serum concentration of TSH was $2.23 \mu \mathrm{IU} / \mathrm{mL}$ (range $0.01-97.18 \mu \mathrm{IU} / \mathrm{mL}$ ). The median tumor size was $9 \mathrm{~mm}$ (range 3-64 mm). The numbers of tumors located in the upper portion, middle portion, lower portion, isthmus and whole thyroid were $592(22.0 \%), 1,133$ (42.2\%), 806 (30.0\%), 85 $(3.2 \%)$ and $71(2.6 \%)$, respectively. Right, left and bilateral tumors were detected in 1,211 (45.1\%), 1,059 (39.4\%), and 417 (15.5\%) patients, respectively. Unifocality and ETE- were detected in 2,364 (88.0\%) and 2,420 (90.1\%) cases, respectively. Histological HT was present in 531 patients (19.8\%) (Table 1).

\section{Lymph Node Yield and Lymph Node Ratio}

Overall, the rate of pCLN- was 51.5\%. The median central lymph node yield (CLNY), central lymph node ratio (CLNR), and the number of ipsilateral CLNDs in the pCLN- cohorts, were significantly lower than the pCLN+ cohorts (Table 1). There were 1606 (59.8\%) ipsilateral CLNDs and 1081 (40.2\%) bilateral CLNDs. The median (IQR) CLNY and CLNR in ipsilateral CLNDs were 7 (7) and $0(0.2)$, respectively, and medians in bilateral CLNDs were 11 (9.5) and 0.18 (0.43), respectively. (not shown in tables).

\section{Clinicopathological Characteristics of Patients in the Derivation Group}

There were no significant differences in CLN pathology, sex, age, BMI, TSH, size, location, unifocality, laterality, ETE-, HT, CLND extent, CLNY or CLNR between the derivation and validation groups (Table 2). We found significant differences in sex, age, BMI, TSH, size, location, unifocality, laterality, and ETEbetween the pCLN- and pCLN+ cohorts in the derivation group based on univariate analysis. However, no significant difference was observed for HT $(\mathrm{P}=0.687)$. The number of CLNDs was excluded from our analysis due to collinearity.

Multivariate analysis indicated that age $(\mathrm{P}<0.001)$, sex $(\mathrm{P}<$ $0.001)$, TSH $(\mathrm{P}=0.003)$, size $(\mathrm{P}<0.001)$, location $(\mathrm{P}<0.001)$, laterality $(\mathrm{P}=0.005)$, unifocality $(\mathrm{P}<0.001)$ and ETE- $(\mathrm{P}<$ 0.001 ) were independent predictors of pCLN-. Overall, BMI had little effect on pCLN-, with no statistical significance was found $(\mathrm{P}=0.781)$ (Table 3).

\section{Prediction Models}

Based on multivariate logistic regression analysis of the derivation group, we developed a prediction model for pCLN-.

$$
\begin{aligned}
& \text { Model : sex + age + TSH + size + location + laterality } \\
& + \text { unifocality + ETE- }
\end{aligned}
$$

The nomogram shows the score of each variable on each scale. Therefore, the probability of pCLN- is determined by the total score of all variables. Inputting the necessary clinicopathological data concisely estimated the probability of pCLN- (Figure 2).

According to this model, the ROC curves of the derivation and validation groups were drawn, and the area under the curve (AUC) was 0.784 (95\% CI 0.763-0.804) in the derivation group and 0.787 (95\% CI 0.756-0.818) in the validation group. The Cindexes of the derivation and validation groups were both 0.783 , which demonstrated good discriminative ability. The calibration plot revealed excellent agreement between the predictions and actual observations (Figure 3). These results showed that this nomogram had good efficacy in predicting the probability of pCLN-. DCA was performed in the validation group. The results suggested that when the threshold probability was approximately $10 \%$ to $90 \%$, compared to a "treat all" or "treat none" strategy, this nomogram yielded a greater net benefit, which indicated that this nomogram had good clinical value (Figure 4).

\section{Follow-up Status}

We followed 2,497 patients (follow-up rate 92.9\%) after the initial surgery until Dec. 2019. For patients without regular evaluations, DFS was based on the time of their last evaluation. The median follow-up period was 30 months (range 3-83 months). Among all 2,497 patients, 2,350 (94.1\%) and 1,475 (59.1\%) patients received TSH suppression and RAI therapy, respectively. Twenty-one $(0.8 \%)$ patients experienced recurrence, and $3(0.1 \%)$ died ( 1 due to asphyxia caused by tracheal metastasis and 2 due to other nontumorous causes). The survival curves plotted according to the Kaplan-Meier method showed that the DFS rate of the 2,497 patients was $99.2 \%$. There was no significant difference in the DFS rate between the pCLNand pCLN+ cohorts ( $99.4 \%$ vs. $98.9 \%, \mathrm{P}=0.126$ ) (Figure 5). We also found significant differences in Tg levels and cervical ultrasound findings between recurrence and nonrecurrence patients (both $\mathrm{P}<0.001$ ), which revealed the importance of periodic monitoring of serum $\mathrm{Tg}$ levels and cervical ultrasound.(not shown in tables).

\section{DISCUSSION}

The present study indicated that for cNO PTC, sex, age, TSH, size, location, unifocality, laterality, and ETE- were independent predictors of pCLN- but BMI and HT were not predictive.

The pCLN- cohorts had significantly lower CLNY and CLNR. This result was primarily because once the presence of CLNM was confirmed on intraoperative frozen section biopsy, prophylactic CLND was changed to therapeutic CLND, and the extent of CLND was further expanded.

The morbidity of PTC is higher in females than males, but the incidence of CLNM is lower $(2,18,19)$. The present study supports these observations, and there was a clear tendency for females to present pCLN- (OR 2.069, $\mathrm{P}<0.001)$. Age is an important basis for TNM staging, and it is one of the factors of many classic prognostic systems: AMES [age, metastasis (distance), extent, size], AGES (age, sex, extent, size), and MACIS [metastasis (distance), age, completeness of surgery, invasiveness, size], which suggests that age is closely related to the biological behavior of PTC. Prognosis and staging may be poorer with increasing age $(6,20,21)$. We found that older patients (age $>45$ years) generally had a significantly higher incidence of pCLN- $(22,23)$, especially patients over 65 years old, due to the susceptibility of older patients to lateral or distant metastasis $(24,25)$. Although no significant difference in pCLNpossibility was found among the three groups in young patients 
TABLE 1 | Clinicopathological characteristics of all patients (derivation + validation groups).

\begin{tabular}{|c|c|c|c|c|c|c|c|}
\hline Characteristics & $\begin{array}{l}\text { Total } \\
2687\end{array}$ & & $\begin{array}{c}\text { pCLN- } \\
1383\end{array}$ & & $\begin{array}{c}\text { pCLN+ } \\
1304\end{array}$ & & $\begin{array}{c}P \text { value } \\
-\end{array}$ \\
\hline Sex & & & & & & & $<0.001$ \\
\hline Male & 684 & $25.5 \%$ & 283 & $20.5 \%$ & 401 & $30.8 \%$ & \\
\hline Female & 2003 & $74.5 \%$ & 1100 & $79.5 \%$ & 903 & $69.2 \%$ & \\
\hline Age Years, Median (IQR) & $43(18)$ & & $45(17)$ & & $41(17)$ & & $<0.001$ \\
\hline$(18,25]$ & 144 & $5.4 \%$ & 48 & $3.5 \%$ & 96 & $7.4 \%$ & \\
\hline$(25,35]$ & 654 & $24.3 \%$ & 269 & $19.5 \%$ & 385 & $29.5 \%$ & \\
\hline$(35,45]$ & 744 & $27.7 \%$ & 375 & $27.1 \%$ & 369 & $28.3 \%$ & \\
\hline$(45,55]$ & 722 & $26.9 \%$ & 428 & $30.9 \%$ & 294 & $22.5 \%$ & \\
\hline$(55,65]$ & 316 & $11.8 \%$ & 192 & $13.9 \%$ & 124 & $9.5 \%$ & \\
\hline$>65$ & 107 & $4.0 \%$ & 71 & $5.1 \%$ & 36 & $2.8 \%$ & \\
\hline BMI [Median (IQR)] & $23.07(4.43)$ & & 22.89 (4.49) & & $23.34(4.41)$ & & 0.039 \\
\hline$<18.5$ & 121 & $4.5 \%$ & 58 & $4.2 \%$ & 63 & $4.8 \%$ & \\
\hline$[18.5,23)$ & 1189 & $44.3 \%$ & 649 & $46.9 \%$ & 540 & $41.4 \%$ & \\
\hline$[23,25)$ & 607 & $22.6 \%$ & 296 & $21.4 \%$ & 311 & $23.8 \%$ & \\
\hline$\geq 25$ & 770 & $28.7 \%$ & 380 & $27.5 \%$ & 390 & $29.9 \%$ & \\
\hline TSH $\mu$ IU/mL, Median (IQR) & $2.23(1.96)$ & & $2.12(1.92)$ & & $2.35(1.97)$ & & 0.005 \\
\hline$\leq 1$ & 319 & $11.9 \%$ & 178 & $12.9 \%$ & 141 & $10.8 \%$ & \\
\hline$(1,2]$ & 837 & $31.1 \%$ & 466 & $33.7 \%$ & 371 & $28.5 \%$ & \\
\hline$(2,3]$ & 679 & $25.3 \%$ & 333 & $24.1 \%$ & 346 & $26.5 \%$ & \\
\hline$(3,4]$ & 414 & $15.4 \%$ & 188 & $13.6 \%$ & 226 & $17.3 \%$ & \\
\hline$(4,5]$ & 204 & $7.6 \%$ & 99 & $7.2 \%$ & 105 & $8.1 \%$ & \\
\hline$>5$ & 234 & $8.7 \%$ & 119 & $8.6 \%$ & 115 & $8.8 \%$ & \\
\hline Size mm, Median (IQR) & $9(8)$ & & $8(5)$ & & $12(10)$ & & $<0.001$ \\
\hline$\leq 5$ & 337 & $12.5 \%$ & 242 & $17.5 \%$ & 95 & $7.3 \%$ & \\
\hline$(5,10]$ & 1266 & $47.1 \%$ & 775 & $56.0 \%$ & 491 & $37.7 \%$ & \\
\hline$(10,15]$ & 527 & $19.6 \%$ & 206 & $14.9 \%$ & 321 & $24.6 \%$ & \\
\hline$(15,20]$ & 261 & $9.7 \%$ & 86 & $6.2 \%$ & 175 & $13.4 \%$ & \\
\hline$(20,25]$ & 116 & $4.3 \%$ & 39 & $2.8 \%$ & 77 & $5.9 \%$ & \\
\hline$(25,30]$ & 71 & $2.6 \%$ & 15 & $1.1 \%$ & 56 & $4.3 \%$ & \\
\hline$>30$ & 109 & $4.1 \%$ & 20 & $1.4 \%$ & 89 & $6.8 \%$ & \\
\hline Location & & & & & & & $<0.001$ \\
\hline Upper & 592 & $22.0 \%$ & 267 & $19.3 \%$ & 325 & $24.9 \%$ & \\
\hline Middle & 1133 & $42.2 \%$ & 762 & $55.1 \%$ & 371 & $28.5 \%$ & \\
\hline Lower & 806 & $30.0 \%$ & 313 & $22.6 \%$ & 493 & $37.8 \%$ & \\
\hline Isthmus & 85 & $3.2 \%$ & 29 & $2.1 \%$ & 56 & $4.3 \%$ & \\
\hline Whole & 71 & $2.6 \%$ & 12 & $0.9 \%$ & 59 & $4.5 \%$ & \\
\hline Laterality & & & & & & & $<0.001$ \\
\hline Left & 1059 & $39.4 \%$ & 583 & $42.2 \%$ & 476 & $36.5 \%$ & \\
\hline Right & 1211 & $45.1 \%$ & 644 & $46.6 \%$ & 567 & $43.5 \%$ & \\
\hline Bilateral & 417 & $15.5 \%$ & 156 & $11.3 \%$ & 261 & $20.0 \%$ & \\
\hline Unifocality & & & & & & & $<0.001$ \\
\hline No & 323 & $12.0 \%$ & 83 & $6.0 \%$ & 240 & $18.4 \%$ & \\
\hline Yes & 2364 & $88.0 \%$ & 1300 & $94.0 \%$ & 1064 & $81.6 \%$ & \\
\hline ETE- & & & & & & & $<0.001$ \\
\hline No & 267 & $9.9 \%$ & 72 & $5.2 \%$ & 195 & $15.0 \%$ & \\
\hline Yes & 2420 & $90.1 \%$ & 1311 & $94.8 \%$ & 1109 & $85.0 \%$ & \\
\hline HT & & & & & & & 0.367 \\
\hline No & 2156 & $80.2 \%$ & 1119 & $80.9 \%$ & 1037 & $79.5 \%$ & \\
\hline Yes & 531 & $19.8 \%$ & 264 & $19.1 \%$ & 267 & $20.5 \%$ & \\
\hline CLND extent & & & & & & & $<0.001$ \\
\hline Ipsilateral & 1606 & $59.8 \%$ & 1041 & $75.3 \%$ & 565 & $43.3 \%$ & \\
\hline Bilateral & 1081 & $40.2 \%$ & 342 & $24.7 \%$ & 739 & $56.7 \%$ & \\
\hline CLNY, Median (IQR) & $9(8)$ & & $8(8)$ & & $10(9)$ & & $<0.001$ \\
\hline CLNR, Median (IQR) & $0.0(0.3)$ & & $0.0(0.0)$ & & $0.3(0.3)$ & & $<0.001$ \\
\hline
\end{tabular}

(age $\leq 45$ years), patients aged 35 to 45 scored higher on the nomogram scale.

TSH regulates the proliferation and function of thyrocytes, and TSH suppression is an important aspect of the comprehensive treatment for PTC. Many studies showed that TSH was associated with tumorigenesis in PTC $(26,27)$.
An increase in TSH may promote tumor progression, and a higher TSH level is a risk factor for PTC and lymph node metastasis $(28,29)$. Patients on levothyroxine or antithyroid drugs preoperatively were excluded from the present study, and we further divided the TSH plasma concentrations into 6 ranges. We found that in the 3 ranges of $\mathrm{TSH} \leq 3 \mu \mathrm{IU} / \mathrm{mL}$, the 
TABLE 2 | Difference between the derivation and validation data groups.

\begin{tabular}{|c|c|c|c|c|c|c|}
\hline Characteristics & $\begin{array}{l}\text { Total } \\
2687\end{array}$ & $\begin{array}{c}\text { Derivation Group } \\
1881\end{array}$ & & $\begin{array}{c}\text { Validation Group } \\
806\end{array}$ & & $\begin{array}{c}\text { P Value } \\
-\end{array}$ \\
\hline Pathology & & & & & & 0.407 \\
\hline pCLN- & 1383 & 978 & $52.0 \%$ & 405 & $50.2 \%$ & \\
\hline $\mathrm{pCLN}+$ & 1304 & 903 & $48.0 \%$ & 401 & $49.8 \%$ & \\
\hline Sex & & & & & & 0.712 \\
\hline Male & 684 & 475 & $25.3 \%$ & 209 & $25.9 \%$ & \\
\hline Female & 2003 & 1406 & $74.7 \%$ & 597 & $74.1 \%$ & \\
\hline Age Years, Median (IQR) & $43(18)$ & $43(18)$ & & $43(18)$ & & 0.493 \\
\hline$(18,25]$ & 144 & 103 & $5.5 \%$ & 41 & $5.1 \%$ & \\
\hline$(25,35]$ & 654 & 448 & $23.8 \%$ & 206 & $25.6 \%$ & \\
\hline$(35,45]$ & 744 & 533 & $28.3 \%$ & 211 & $26.2 \%$ & \\
\hline$(45,55]$ & 722 & 491 & $26.1 \%$ & 231 & $28.7 \%$ & \\
\hline$(55,65]$ & 316 & 230 & $12.2 \%$ & 86 & $10.7 \%$ & \\
\hline$>65$ & 107 & 76 & $4.0 \%$ & 31 & $3.8 \%$ & \\
\hline BMI [Median (IQR)] & $23.07(4.43)$ & $23.05(4.30)$ & & $23.14(4.77)$ & & 0.515 \\
\hline$>18.5$ & 121 & 80 & $4.3 \%$ & 41 & $5.1 \%$ & \\
\hline$[18.5,23)$ & 1189 & 842 & $44.8 \%$ & 347 & $43.1 \%$ & \\
\hline$[23,25)$ & 607 & 431 & $22.9 \%$ & 176 & $21.8 \%$ & \\
\hline$\geq 25$ & 770 & 528 & $28.1 \%$ & 242 & $30.0 \%$ & \\
\hline TSH $\mu \mathrm{IU} / \mathrm{mL}$, Median (IQR) & $2.23(1.96)$ & $2.24(1.95)$ & & $2.22(2)$ & & 0.287 \\
\hline$\leq 1$ & 319 & 228 & $12.1 \%$ & 91 & $11.3 \%$ & \\
\hline$(1,2]$ & 837 & 580 & $30.8 \%$ & 257 & $31.9 \%$ & \\
\hline$(2,3]$ & 679 & 476 & $25.3 \%$ & 203 & $25.2 \%$ & \\
\hline$(3,4]$ & 414 & 305 & $16.2 \%$ & 109 & $13.5 \%$ & \\
\hline$(4,5]$ & 204 & 132 & $7.0 \%$ & 72 & $8.9 \%$ & \\
\hline$>5$ & 234 & 160 & $8.5 \%$ & 74 & $9.2 \%$ & \\
\hline Size mm, Median (IQR) & $9(8)$ & $9(7)$ & & $10(8)$ & & 0.781 \\
\hline$\leq 5$ & 337 & 240 & $12.8 \%$ & 97 & $12.0 \%$ & \\
\hline$(5,10]$ & 1266 & 883 & $46.9 \%$ & 383 & $47.5 \%$ & \\
\hline$(10,15]$ & 527 & 380 & $20.2 \%$ & 147 & $18.2 \%$ & \\
\hline$(15,20]$ & 261 & 177 & $9.4 \%$ & 84 & $10.4 \%$ & \\
\hline$(20,25]$ & 116 & 77 & $4.1 \%$ & 39 & $4.8 \%$ & \\
\hline$(25,30]$ & 71 & 47 & $2.5 \%$ & 24 & $3.0 \%$ & \\
\hline$>30$ & 109 & 77 & $4.1 \%$ & 32 & $4.0 \%$ & \\
\hline Location & & & & & & 0.07 \\
\hline Upper & 592 & 411 & $21.9 \%$ & 181 & $22.5 \%$ & \\
\hline Middle & 1133 & 822 & $43.7 \%$ & 311 & $38.6 \%$ & \\
\hline Lower & 806 & 536 & $28.5 \%$ & 270 & $33.5 \%$ & \\
\hline Isthmus & 85 & 60 & $3.2 \%$ & 25 & $3.1 \%$ & \\
\hline Whole & 71 & 52 & $2.8 \%$ & 19 & $2.4 \%$ & \\
\hline Laterality & & & & & & 0.125 \\
\hline Left & 1059 & 765 & $40.7 \%$ & 294 & $36.5 \%$ & \\
\hline Right & 1211 & 830 & $44.1 \%$ & 381 & $47.3 \%$ & \\
\hline Bilateral & 417 & 286 & $15.2 \%$ & 131 & $16.3 \%$ & \\
\hline Unifocality & & & & & & 0.709 \\
\hline No & 323 & 229 & $12.2 \%$ & 94 & $11.7 \%$ & \\
\hline Yes & 2364 & 1652 & $87.8 \%$ & 712 & $88.3 \%$ & \\
\hline ETE- & & & & & & 0.99 \\
\hline No & 267 & 187 & $9.9 \%$ & 80 & $9.9 \%$ & \\
\hline Yes & 2420 & 1694 & $90.1 \%$ & 726 & $90.1 \%$ & \\
\hline HT & & & & & & 0.651 \\
\hline No & 2156 & 1505 & $80.0 \%$ & 651 & $80.8 \%$ & \\
\hline Yes & 531 & 376 & $20.0 \%$ & 155 & $19.2 \%$ & \\
\hline CLND extent & & & & & & 0.151 \\
\hline Ipsilateral & 1606 & 1141 & $60.7 \%$ & 465 & $57.7 \%$ & \\
\hline Bilateral & 1081 & 740 & $39.3 \%$ & 341 & $42.3 \%$ & \\
\hline CLNY, Median (IQR) & $9(8)$ & $8(8)$ & & $9(8)$ & & 0.445 \\
\hline CLNR, Median (IQR) & $0.0(0.3)$ & $0.0(0.3)$ & & $0.0(0.3)$ & & 0.417 \\
\hline
\end{tabular}

probability of PCLN- increased with decreasing TSH levels, and the opposite was true in the other 3 ranges of TSH $>3 \mu \mathrm{IU} / \mathrm{mL}$. This result suggests that the level of TSH and the probability of PCLN- are not simple linear correlations.
Size reflects solid tumor staging, and it is related to many aspects, such as treatment and prognosis. PTC with a size $\leq 10$ $\mathrm{mm}$ is defined as papillary thyroid microcarcinoma (PTMC), and most of these cases tend to exhibit less CLNM and a better 
TABLE 3 | Univariate and multivariate analysis of $\mathrm{PCLN}$ - in the derivation group.

\begin{tabular}{|c|c|c|c|c|c|c|c|c|c|c|}
\hline Characteristics & $\begin{array}{l}\text { Total } \\
1881\end{array}$ & & $\begin{array}{c}\text { pCLN- } \\
978\end{array}$ & & $\begin{array}{c}\text { pCLN+ } \\
903\end{array}$ & & $\begin{array}{c}\text { Univariate analysis } \\
\text { OR }(95 \% \mathrm{Cl})\end{array}$ & P Value & $\begin{array}{c}\text { Multivariate analysis } \\
\text { OR }(95 \% \mathrm{Cl})\end{array}$ & P Value \\
\hline Sex & & & & & & & & $<0.001$ & & $<0.001$ \\
\hline Male & 475 & $25.3 \%$ & 193 & $19.7 \%$ & 282 & $31.2 \%$ & 1 (reference) & & 1 (reference) & \\
\hline Female & 1406 & $74.7 \%$ & 785 & $80.3 \%$ & 621 & $68.8 \%$ & $1.847(1.495-2.281)$ & & 2.069 (1.608-2.663) & \\
\hline Age Years, Median (IQR) & $43(18)$ & & $45(16)$ & & $41(17)$ & & & $<0.001$ & & $<0.001$ \\
\hline$(18,25]$ & 103 & $5.5 \%$ & 40 & $4.1 \%$ & 63 & $7.0 \%$ & 1 (reference) & & 1 (reference) & \\
\hline$(25,35]$ & 448 & $23.8 \%$ & 184 & $18.8 \%$ & 264 & $29.2 \%$ & $1.098(0.708-1.702)$ & & 0 & \\
\hline$(35,45]$ & 533 & $28.3 \%$ & 271 & $27.7 \%$ & 262 & $29.0 \%$ & $1.629(1.059-2.507)$ & & $0.884(0.534-1.465)$ & \\
\hline$(45,55]$ & 491 & $26.1 \%$ & 289 & $29.6 \%$ & 202 & $22.4 \%$ & $2.253(1.458-3.482)$ & & $1.212(0.735-2.000)$ & \\
\hline$(55,65]$ & 230 & $12.2 \%$ & 143 & $14.6 \%$ & 87 & $9.6 \%$ & $2.589(1.606-4.173)$ & & $2.074(1.251-3.440)$ & \\
\hline$>65$ & 76 & $4.0 \%$ & 51 & $5.2 \%$ & 25 & $2.8 \%$ & $3.213(1.726-5.98)$ & & $2.360(1.357-4.104)$ & \\
\hline BMI Median (IQR) & $\begin{array}{l}23.05 \\
(4.30)\end{array}$ & & $\begin{array}{l}22.86 \\
(4.27)\end{array}$ & & $\begin{array}{l}23.37 \\
(4.17)\end{array}$ & & & 0.041 & & 0.781 \\
\hline$<18.5$ & 80 & $4.3 \%$ & 46 & $4.7 \%$ & 34 & $3.8 \%$ & 1 (reference) & & 1 (reference) & \\
\hline$[18.5,23)$ & 842 & $44.8 \%$ & 464 & $47.4 \%$ & 378 & $41.9 \%$ & $0.907(0.571-1.442)$ & & $1.052(0.626-1.766)$ & \\
\hline$[23,25)$ & 431 & $22.9 \%$ & 213 & $21.8 \%$ & 218 & $24.1 \%$ & $0.722(0.446-1.169)$ & & $0.909(0.524-1.577)$ & \\
\hline$\geq 25$ & 528 & $28.1 \%$ & 255 & $26.1 \%$ & 273 & $30.2 \%$ & $0.690(0.429-1.11)$ & & $0.991(0.574-1.712)$ & \\
\hline $\begin{array}{l}\text { TSH } \mu \mid U / m L, \text { Median } \\
\text { (IQR) }\end{array}$ & $2.24(1.95)$ & & $2.12(1.90)$ & & $2.36(1.99)$ & & & 0.012 & & 0.003 \\
\hline$\leq 1$ & 228 & $12.1 \%$ & 129 & $13.2 \%$ & 99 & $11.0 \%$ & 1 (reference) & & 1 (reference) & \\
\hline$(1,2]$ & 580 & $30.8 \%$ & 330 & $33.7 \%$ & 250 & $27.7 \%$ & $1.013(0.744-1.380)$ & & $0.931(0.654-1.325)$ & \\
\hline$(2,3]$ & 476 & $25.3 \%$ & 235 & $24.0 \%$ & 241 & $26.7 \%$ & $0.748(0.545-1.028)$ & & $0.686(0.477-0.986)$ & \\
\hline$(3,4]$ & 305 & $16.2 \%$ & 139 & $14.2 \%$ & 166 & $18.4 \%$ & $0.643(0.455-0.908)$ & & $0.526(0.353-0.783)$ & \\
\hline$(4,5]$ & 132 & $7.0 \%$ & 64 & $6.5 \%$ & 68 & $7.5 \%$ & $0.722(0.470-1.11)$ & & 0.599 (0.365-0.983) & \\
\hline$>5$ & 160 & $8.5 \%$ & 81 & $8.3 \%$ & 79 & $8.7 \%$ & $0.787(0.524-1.181)$ & & $0.649(0.405-1.04)$ & \\
\hline Size mm, Median (IQR) & $9(7)$ & & $8(5)$ & & $12(9)$ & & & $<0.001$ & & $<0.001$ \\
\hline$\leq 5$ & 240 & $12.8 \%$ & 176 & $18.0 \%$ & 64 & $7.1 \%$ & 1 (reference) & & 1 (reference) & \\
\hline$(5,10]$ & 883 & $46.9 \%$ & 538 & $55.0 \%$ & 345 & $38.2 \%$ & $0.567(0.413-0.778)$ & & $0.544(0.385-0.768)$ & \\
\hline$(10,15]$ & 380 & $20.2 \%$ & 151 & $15.4 \%$ & 229 & $25.4 \%$ & $0.240(0.169-0.341)$ & & $0.255(0.173-0.376)$ & \\
\hline$(15,20]$ & 177 & $9.4 \%$ & 58 & $5.9 \%$ & 119 & $13.2 \%$ & $0.177(0.116-0.271)$ & & $0.217(0.136-0.345)$ & \\
\hline$(20,25]$ & 77 & $4.1 \%$ & 28 & $2.9 \%$ & 49 & $5.4 \%$ & $0.208(0.120-0.358)$ & & $0.201(0.110-0.370)$ & \\
\hline$(25,30]$ & 47 & $2.5 \%$ & 11 & $1.1 \%$ & 36 & $4.0 \%$ & $0.111(0.053-0.231)$ & & $0.117(0.053-0.258)$ & \\
\hline$>30$ & 77 & $4.1 \%$ & 16 & $1.6 \%$ & 61 & $6.8 \%$ & $0.095(0.051-0.177)$ & & $0.187(0.074-0.474)$ & \\
\hline Location & & & & & & & & $<0.001$ & & $<0.001$ \\
\hline Upper & 411 & $21.9 \%$ & 189 & $19.3 \%$ & 222 & $24.6 \%$ & $0.419(0.329-0.534)$ & & $0.455(0.348-0.595)$ & \\
\hline Middle & 822 & $43.7 \%$ & 551 & $56.3 \%$ & 271 & $30.0 \%$ & 1 (reference) & & 1 (reference) & \\
\hline Lower & 536 & $28.5 \%$ & 208 & $21.3 \%$ & 328 & $36.3 \%$ & $0.312(0.249-0.391)$ & & $0.304(0.236-0.392)$ & \\
\hline Isthmus & 60 & $3.2 \%$ & 21 & $2.1 \%$ & 39 & $4.3 \%$ & $0.265(0.153-0.459)$ & & $0.337(0.179-0.635)$ & \\
\hline Whole & 52 & $2.8 \%$ & 9 & $0.9 \%$ & 43 & $4.8 \%$ & $0.103(0.049-0.214)$ & & $0.219(0.071-0.671)$ & \\
\hline Laterality & & & & & & & & $<0.001$ & & 0.005 \\
\hline Left & 765 & $40.7 \%$ & 417 & $42.6 \%$ & 348 & $38.5 \%$ & 1 (reference) & & 1 (reference) & \\
\hline Right & 830 & $44.1 \%$ & 461 & $47.1 \%$ & 369 & $40.9 \%$ & $1.043(0.856-1.270)$ & & $1.082(0.864-1.356)$ & \\
\hline Bilateral & 286 & $15.2 \%$ & 100 & $10.2 \%$ & 186 & $20.6 \%$ & $0.449(0.339-0.595)$ & & $0.629(0.452-0.875)$ & \\
\hline Unifocality & & & & & & & & $<0.001$ & & $<0.001$ \\
\hline No & 229 & $12.2 \%$ & 58 & $5.9 \%$ & 171 & $18.9 \%$ & 0.270 (0.197-0.369) & & $0.294(0.206-0.419)$ & \\
\hline Yes & 1652 & $87.8 \%$ & 920 & $94.1 \%$ & 732 & $81.1 \%$ & 1 (reference) & & 1 (reference) & \\
\hline ETE- & & & & & & & & $<0.001$ & & $<0.001$ \\
\hline No & 187 & $9.9 \%$ & 54 & $5.5 \%$ & 133 & $14.7 \%$ & 1 (reference) & & 1 (reference) & \\
\hline Yes & 1694 & $90.1 \%$ & 924 & $94.5 \%$ & 770 & $85.3 \%$ & $2.956(2.124-4.113)$ & & 2.235 (1.520-3.288) & \\
\hline HT & & & & & & & & 0.687 & & - \\
\hline No & 1505 & $80.0 \%$ & 786 & $80.4 \%$ & 719 & $79.6 \%$ & 1 (reference) & & & \\
\hline Yes & 376 & $20.0 \%$ & 192 & $19.6 \%$ & 184 & $20.4 \%$ & $0.955(0.761-1.197)$ & & & \\
\hline
\end{tabular}

prognosis, which suggests indolent behavior. Yu et al. included 11,845 PTMC patients in the SEER database and found that the LNM incidence of PTMC was $12 \%$. Similarly, Ito et al. revealed an LNM incidence of $<5 \%$ based on 5-10 years of PTMC data, and all new metastases appeared in the lateral compartment instead of the central compartment $(5,30,31)$. Compared to PTC, which has an incidence range of $30-90 \%$, PTMC is less prone to LN involvement, which suggests that PTC of a small size exhibits less nodal invasion $(16,32)$. We found that even for
PTMC, compared to size $>5$ but $\leq 10 \mathrm{~mm}$, size $\leq 5 \mathrm{~mm}$ significantly increased the possibility of pCLN-, which obtained the highest score on the nomogram. Notably, the tumor size almost linearly negatively correlated with the probability of pCLN- except for the size $>25$ but $\leq 30 \mathrm{~mm}$ group. The number of patients with tumor sizes between 25 and $30 \mathrm{~mm}$ in this study was the lowest(71 in total and 47 in the derivation group). More detailed studies are needed to determine whether this result was due to the decrease in CLNM when the 


\section{Points}

\section{Sex}

Age

TSH

Size

\section{Location}

Laterality

Unifocality

ETE-

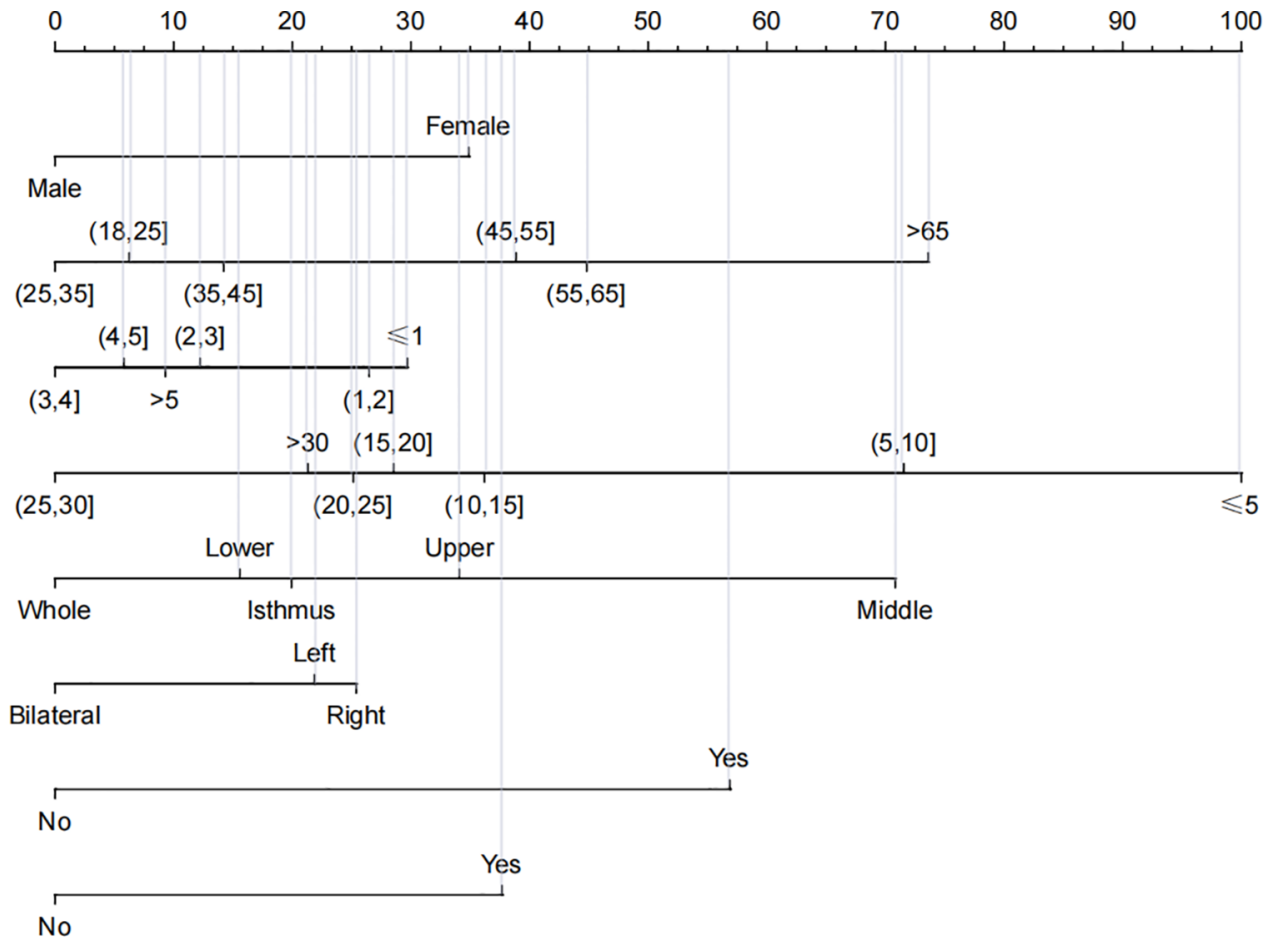

Total Points

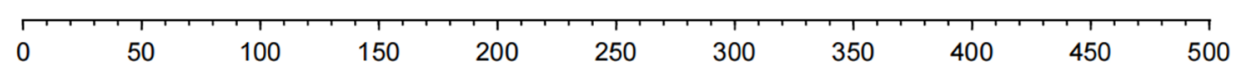

Possibility of pCLN-

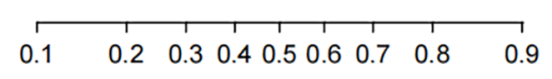

FIGURE 2 | Nomogram for prediction of pCLN-. A line is drawn straight up to the point axis that corresponds with each patient variable to obtain the points. The sum of these points is located on the total score points axis. A line is drawn downwards to the risk axis to determine the possibility of pCLN-.

tumor developed to a certain critical size or to the bias caused by an insufficient sample size.

Lymphatic vessels originating at the thyroid form a subcapsular lymphatic network on the surface of the gland. In addition to the superior and inferior thyroid arteries and veins, some lymphatic vessels run along the middle thyroid vein, and some do not accompany the blood vessels. This network is how thyroid-centered lymphatic vessels connect to the surrounding cervical lymph nodes. Different thyroid gland locations have different lymphatic drainage paths. Lymphatic drainage of the upper thyroid is primarily collected by the lymphatic vessels accompanying the superior thyroid artery, with some drainage flowing from the central to the lateral compartment, and some drainage flowing into the venous system through the lymph nodes of the lateral compartment. Lymphatic drainage of the lower thyroid is primarily collected by the lymphatic vessels accompanying the inferior thyroid artery and flowing into the lateral lymph nodes through the CLNs (especially the paratracheal lymph nodes) and ultimately into the venous system. Lymphatic drainage from the isthmus of the thyroid primarily descends to the mediastinal lymph nodes $(33,34)$.
We found that the middle portion was the most common location for PTC (43.7\%) and the location with the highest likelihood of pCLN-. This result may be explained by the more complex and crisscrossing lymphatic drainage pathways in the middle portion, and drainage of this region may be carried up or down before proceeding through the upper or lower lymphatic vessels to the corresponding lymph nodes. Therefore, middle tumors progress more slowly than tumors in other portions. There is also a lateral drainage pathway in the middle of the thyroid that passes through the deep surface of the cervical sheath. However, these hypotheses emphasize the need for more accurate anatomical studies to understand the patterns of metastatic spread in PTC. Previous studies reported that an upper location was a risk factor for LLNM, and tumors located in the isthmus and lower portion were more likely to result in CLNM $(35,36)$. Consistent with these studies, we found that the probability of pCLN- in upper tumors was second only to middle tumors. The probability of pCLN- in isthmic and lower tumors was reduced even further.

Bilaterality or multifocality may develop via intraglandular spread from a single primary tumor or multiple synchronous 
A

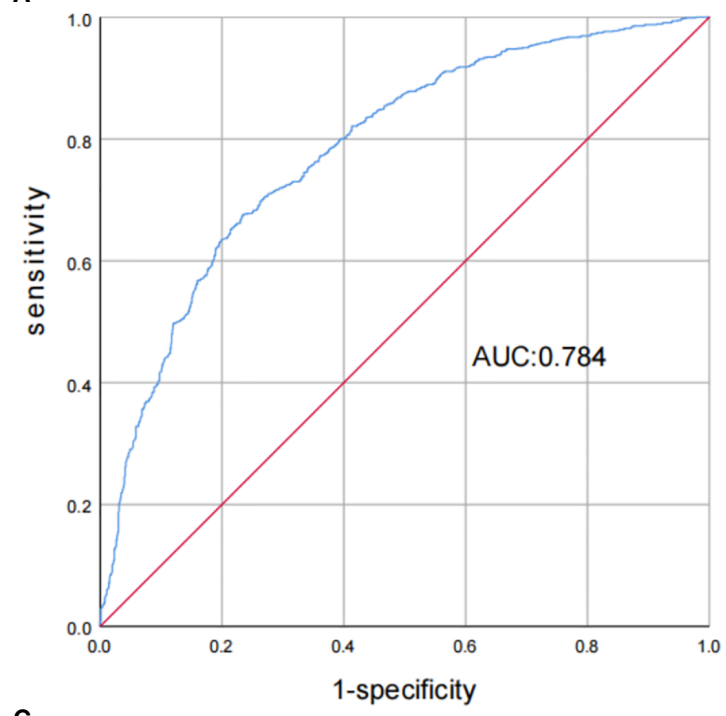

C

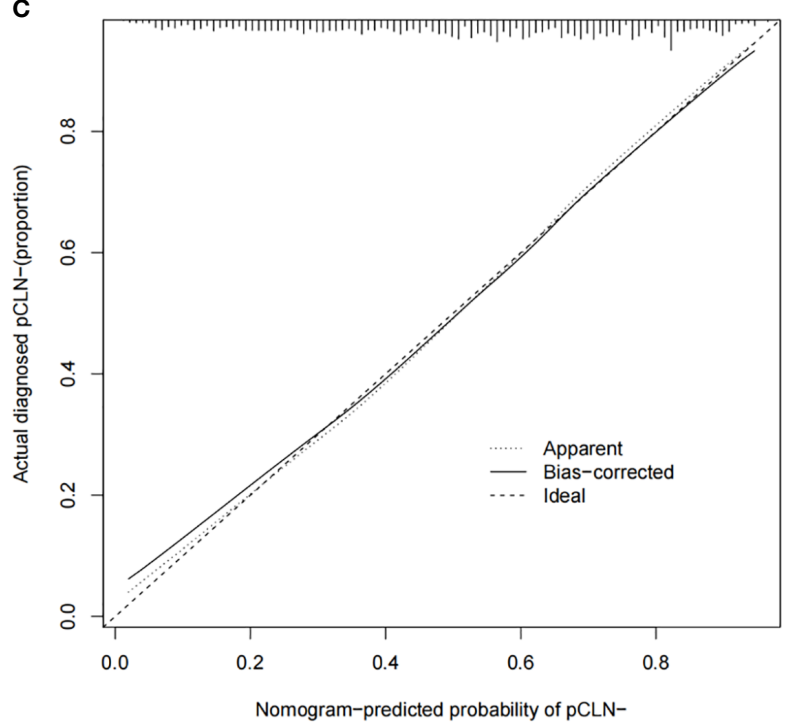

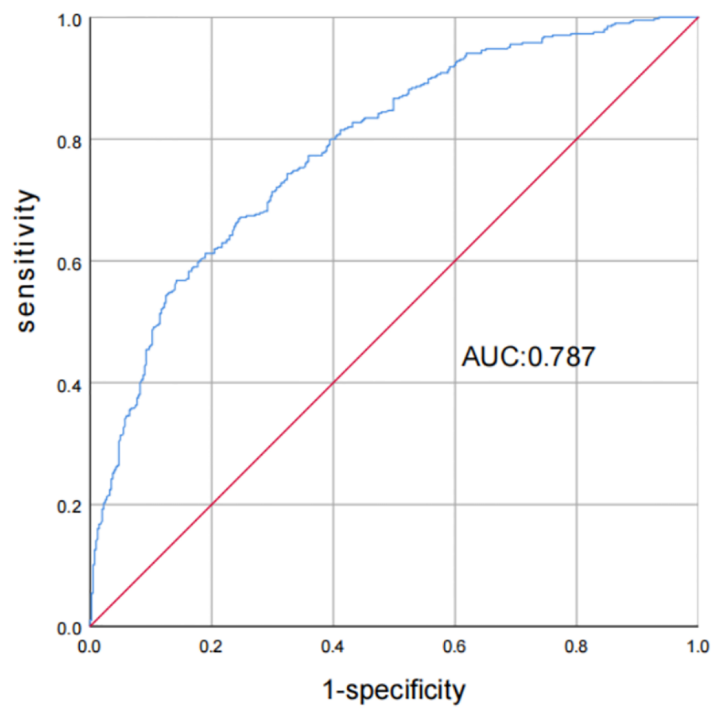

D

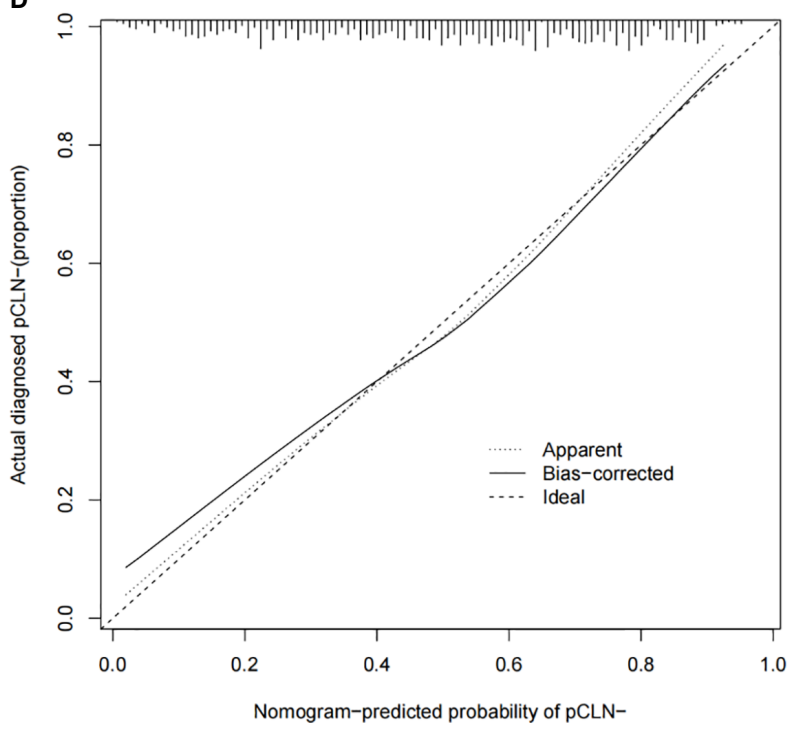

FIGURE 3 | Receiver operating characteristic curves and calibration plot of the model. In the derivation group (A), the sensitivity and specificity were $67.5 \%$ and $76.6 \%$, respectively, with an AUC of $0.784(95 \% \mathrm{Cl} 0.763-0.804)$. In the validation group (B), the sensitivity and specificity were $67.2 \%$ and $75.3 \%$, respectively, with an AUC of 0.787 (95\% Cl $0.756-0.818)$. The calibration plot depicts the calibration of the model in terms of the agreement between the predicted possibility of pCLN- and the observed outcomes of pCLN-. The derivation group (C) and the validation group (D) indicated good prediction.

primary tumors (37). The invasiveness of unilateral and unifocal PTC is weaker than bilateral and multifocal PTC $(38,39)$. The present study also suggested that CLNs were less prone to invasion in unilateral or unifocal PTC. We further found no significant difference in the probability of pCLN- between the left and right lobes. The left and right lobes of the thyroid gland were not completely symmetrical, but the right lobe was generally larger (40).

ETE- refers to tumors confined to the thyroid capsule. It is easier for the tumor to invade lymph nodes through lymphatics on the surface of the capsule after the thyroid capsule is breached $(4,41)$. We also found that ETE- was separately and independently associated with pCLN- in cNO PTC.
The incidence of being overweight increased synchronously with the morbidity of PTC (42), but the relationship between BMI and PTC is not clear. A study of 11,323,006 adults showed that BMI and waist circumference were associated with PTC, and weight loss was associated with lower PTC morbidity (43). Kang et al. and Ray et al. found that fibroblast growth factor 21 (FGF21) positively correlated with BMI, and leptin played a role as a proinflammatory factor in overweight people. FGF21 and leptin were also involved in the regulation of PTC development, which indicated that BMI was also related to invasion and metastasis in PTC $(44,45)$. The BMI level of the pCLN- cohort in the present study was slightly lower, but there was no significant correlation between BMI and pCLN-, which is 


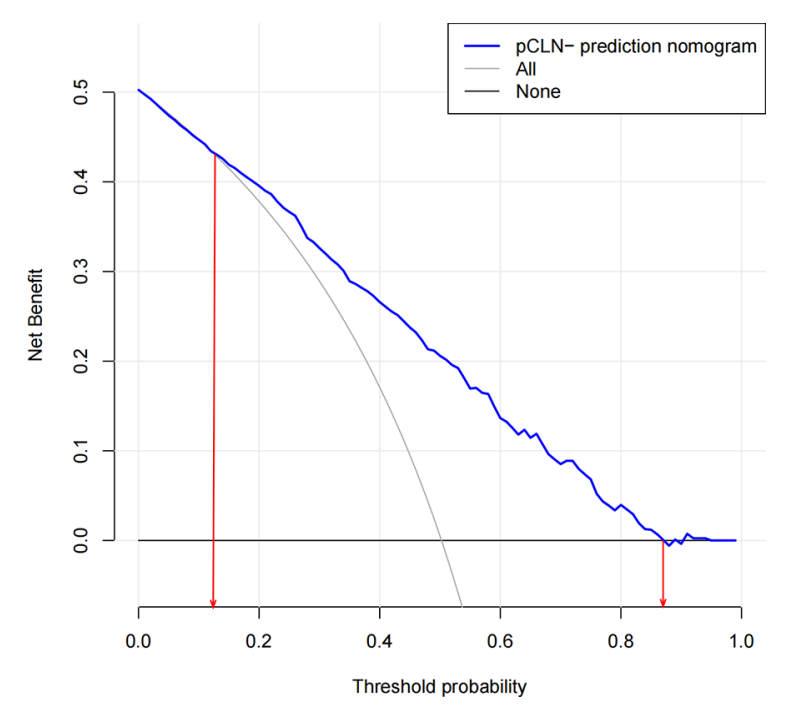

FIGURE 4 | Decision curve analysis for the nomogram(in the validation group). The possibility of an individual is denoted as $\mathrm{Pi}$. When $\mathrm{Pi}$ reaches a certain threshold (denoted as Pt), it is defined as positive, and intervention (not performing CLND) may be taken. Therefore, there are benefits (pros) of intervention for patients with pCLN- and harm (cons) of intervention for patients with pCLN+. There is also the harm (cons) of missed intervention for pCLN+ patients. Pros minus cons is the net benefit. When $\mathrm{Pi}<\mathrm{Pt}$, there is no intervention, and the net benefit is 0 (treat-none). When $\mathrm{Pi}>\mathrm{Pt}$, all patients receive the intervention, and the net benefit is shown by a gray slanted curve (treat-all). Our decision curve indicates that when the threshold probability is approximately $10 \%$ to $90 \%$, the use of this predictive model would accrue greater benefit than a treat-all or treat-none strategy.

consistent with the results obtained by Grani et al. and Paes et al. $(46,47)$. Because being overweight may involve aromatase activation, chronic inflammation and oxidative stress, more molecular biological mechanisms that affect the relationship between BMI, PTC and invasiveness must be explored.

HT is a type of autoimmune thyroiditis that is characterized by an abundance of lymphocytes, and it also known as chronic lymphocyte thyroiditis. The relationship between HT and CLN status in PTC is inconclusive $(48,49)$, and the differences in diagnostic criteria, severity, antibody levels, and the heterogeneity of HT may underlie these controversies. HT was confirmed via postoperative pathology in this study, and we found that HT barely affected CLN status. However, this study did not determine the level of autoantibodies (primarily TPO-Ab and Tg-Ab), and quantitative analysis of the degree of HT before surgery needs further study.

The follow-up results showed that very few patients experienced lymph node recurrence, which confirmed that the lymph node pathologic status, especially in the pCLN- cohort was reliable. Specifically, the derivation basis of the nomogram was reliable. Because the present study was a single-center retrospective study, selection deviation is inevitable. Notably, the lack of external validation is a limitation, and relevant prospective multicenter clinical studies should be performed in the future to evaluate the accuracy of the proposed model. Analysis of potential confounders of future metastasis was not possible due to the relatively short follow-up, which suggests that prolonged follow-up and more data are required in our further work.

There have been many prediction models of CLNM in $\mathrm{cN} 0$ PTC $(50,51)$. However, available prediction models for other patients who present pCLN- and constitute the majority of the study population (the pCLN- rate was $51.5 \%$ in this study) are lacking. In conclusion, we established the first quantified prediction model for pCLN- in cNO PTC. Clinicians can use this nomogram to evaluate patients with PTC based on clinicopathological characteristics. For cN0 PTC, which has a
A

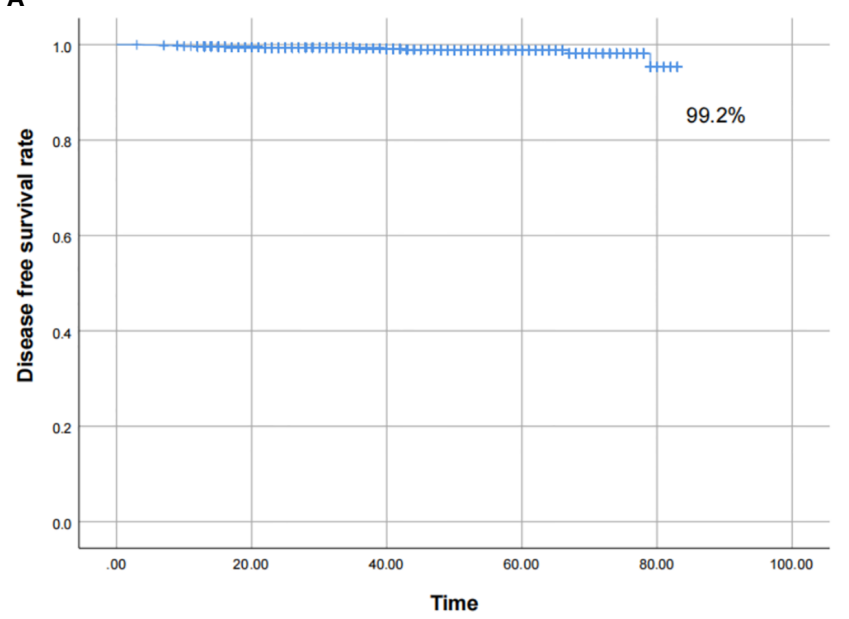

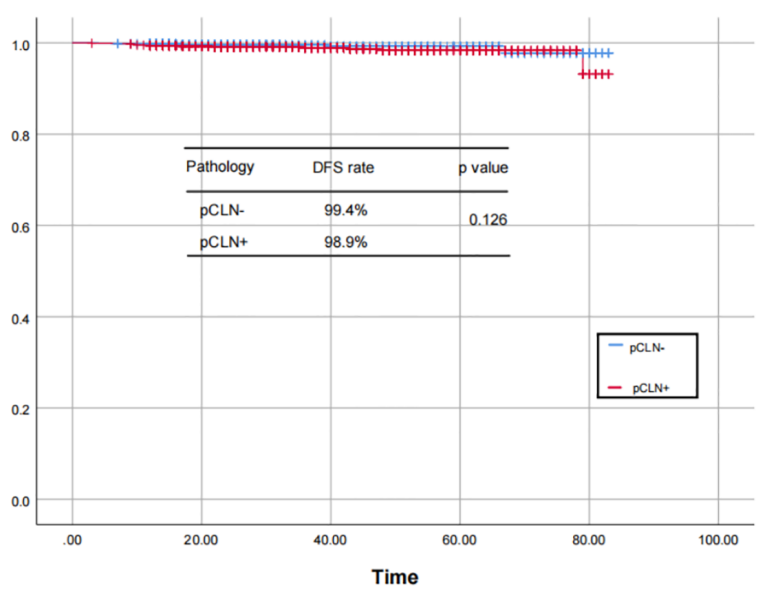

FIGURE 5 | The Kaplan-Meier curves for the 2,497 patients. The curves show that the DFS rate was $99.2 \%$ in 2,497 patients (A), and there was no significant difference in the DFS rate between the PCLN- and PCLN+ cohorts (B). 
high probability of pCLN-, unnecessary prophylactic CLND can may be avoided.

\section{DATA AVAILABILITY STATEMENT}

The original contributions presented in the study are included in the article/supplementary material. Further inquiries can be directed to the corresponding author.

\section{ETHICS STATEMENT}

The studies involving human participants were reviewed and approved by Ethics Committee of the First Affiliated Hospital of Chongqing Medical University. Written informed consent for

\section{REFERENCES}

1. Siegel RL, Miller KD, Jemal A. Cancer Statistics, 2020. CA Cancer J Clin (2020) 70(1):7-30. doi: 10.3322/caac.21590

2. Cabanillas ME, McFadden DG, Durante C. Thyroid Cancer. Lancet (2016) 388(10061):2783-95. doi: 10.1016/S0140-6736(16)30172-6

3. Burgess JR, Tucker P. Incidence Trends for Papillary Thyroid Carcinoma and Their Correlation With Thyroid Surgery and Thyroid Fine-Needle Aspirate Cytology. Thyroid (2006) 16(1):47-53. doi: 10.1089/thy.2006.16.47

4. Goyal N, Pakdaman M, Kamani D, Caragacianu D, Goldenberg D, Randolph GW. Mapping the Distribution of Nodal Metastases in Papillary Thyroid Carcinoma: Where Exactly are the Nodes? Laryngoscope (2017) 127(8):195964. doi: 10.1002/lary.26495

5. Haugen BR, Alexander EK, Bible KC, Doherty GM, Mandel SJ, Nikiforov YE, et al. 2015 American Thyroid Association Management Guidelines for Adult Patients With Thyroid Nodules and Differentiated Thyroid Cancer: The American Thyroid Association Guidelines Task Force on Thyroid Nodules and Differentiated Thyroid Cancer. Thyroid (2016) 26(1):1-133. doi: 10.1089/thy.2015.0020

6. American Thyroid Association Surgery Working G, American Association of Endocrine S and American Academy of O-H, Neck S, American H, Neck S, et al. Consensus Statement on the Terminology and Classification of Central Neck Dissection for Thyroid Cancer. Thyroid (2009) 19(11):1153-8. doi: 10.1089/thy.2009.0159

7. Amin MB, Gress DM. AJCC Cancer Staging Manual. Eight Edition. New York: Springer (2017). doi: 10.1007/s00106-017-0391-3

8. Eun NL, Son EJ, Kim JA, Gweon HM, Kang JH, Youk JH. Comparison of the Diagnostic Performances of Ultrasonography, CT and Fine Needle Aspiration Cytology for the Prediction of Lymph Node Metastasis in Patients With Lymph Node Dissection of Papillary Thyroid Carcinoma: A Retrospective Cohort Study. Int J Surg (2018) 51:145-50. doi: 10.1016/j.ijsu.2017.12.036

9. Rosario PW, de Faria S, Bicalho L, Alves MF, Borges MA, Purisch S, et al. Ultrasonographic Differentiation Between Metastatic and Benign Lymph Nodes in Patients With Papillary Thyroid Carcinoma. J Ultrasound Med (2005) 24(10):1385-9. doi: 10.7863/jum.2005.24.10.1385

10. Hoang JK, Lee WK, Lee M, Johnson D, Farrell S. US Features of Thyroid Malignancy: Pearls and Pitfalls. Radiographics (2007) 27(3):847-60. doi: 10.1148/rg.273065038 discussion 861-845.

11. National Comprehensive Cancer Network. (NCCN) Clinical Practice Guidelines in Oncology, in: Thyroid Carcinoma Version1 (2021). Available at: https://www.nccn.org (Accessed 25 Aug 2021).

12. Roh JL, Park JY, Park CI. Total Thyroidectomy Plus Neck Dissection in Differentiated Papillary Thyroid Carcinoma Patients: Pattern of Nodal Metastasis, Morbidity, Recurrence, and Postoperative Levels of Serum Parathyroid Hormone. Ann Surg (2007) 245(4):604-10. doi: 10.1097/ 01.sla.0000250451.59685.67 participation was not required for this study in accordance with the national legislation and the institutional requirements.

\section{AUTHOR CONTRIBUTIONS}

XJS and XLS designed this study. XJS, LT, DH, YW, PY, ZY, $\mathrm{CD}$, and DW collected the data. XJS analyzed the data. All authors contributed to the article and approved the submitted version.

\section{ACKNOWLEDGMENTS}

The authors appreciate all of the staff involved in the preparation of the study.

13. So YK, Son YI, Hong SD, Seo MY, Beak CH, Jeong HS, et al. Subclinical Lymph Node Metastasis in Papillary Thyroid Microcarcinoma: A Study of 551 Resections. Surgery (2010) 148(3):526-31. doi: 10.1016/j.surg.2010.01.003

14. Popadich A, Levin O, Lee JC, Smooke-Praw S, Ro K, Fazel M, et al. A Multicenter Cohort Study of Total Thyroidectomy and Routine Central Lymph Node Dissection for Cn0 Papillary Thyroid Cancer. Surgery (2011) 150(6):1048-57. doi: 10.1016/j.surg.2011.09.003

15. Kouvaraki MA, Shapiro SE, Fornage BD, Edeiken-Monro BS, Sherman SI, Vassilopoulou-Sellin R, et al. Role of Preoperative Ultrasonography in the Surgical Management of Patients With Thyroid Cancer. Surgery (2003) 134 (6):946-54. doi: 10.1016/s0039-6060(03)00424-0 discussion 954-945.

16. Hall FT, Beasley NJ, Eski SJ, Witterick IJ, Walfish PG, Freeman JL. Predictive Value of Serum Thyroglobulin After Surgery for Thyroid Carcinoma. Laryngoscope (2003) 113(1):77-81. doi: 10.1097/00005537200301000-00014

17. Nascimento C, Borget I, Troalen F, Al Ghuzlan A, Deandreis D, Hartl D, et al. Ultrasensitive Serum Thyroglobulin Measurement is Useful for the Follow-Up of Patients Treated With Total Thyroidectomy Without Radioactive Iodine Ablation. Eur J Endocrinol (2013) 169(5):689-93. doi: 10.1530/EJE-13-0386

18. Smith VA, Sessions RB, Lentsch EJ. Cervical Lymph Node Metastasis and Papillary Thyroid Carcinoma: Does the Compartment Involved Affect Survival? Experience From the SEER Database. J Surg Oncol (2012) 106 (4):357-62. doi: 10.1002/jso.23090

19. Zheng X, Peng C, Gao M, Zhi J, Hou X, Zhao J, et al. Risk Factors for Cervical Lymph Node Metastasis in Papillary Thyroid Microcarcinoma: A Study of 1,587 Patients. Cancer Biol Med (2019) 16(1):121-30. doi: 10.20892/ j.issn.2095-3941.2018.0125

20. Ito Y, Kudo T, Kobayashi K, Miya A, Ichihara K, Miyauchi A. Prognostic Factors for Recurrence of Papillary Thyroid Carcinoma in the Lymph Nodes, Lung, and Bone: Analysis of 5,768 Patients With Average 10-Year Follow-Up. World J Surg (2012) 36(6):1274-8. doi: 10.1007/s00268-012-1423-5

21. Kauffmann RM, Hamner JB, Ituarte PHG, Yim JH. Age Greater Than 60 Years Portends a Worse Prognosis in Patients With Papillary Thyroid Cancer: Should There be Three Age Categories for Staging? BMC Cancer (2018) 18 (1):316. doi: 10.1186/s12885-018-4181-4

22. Vriens MR, Moses W, Weng J, Peng M, Griffin A, Bleyer A, et al. Clinical and Molecular Features of Papillary Thyroid Cancer in Adolescents and Young Adults. Cancer (2011) 117(2):259-67. doi: 10.1002/cncr.25369

23. Jianming L, Jibin L, Linxue Q. Suspicious Ultrasound Characteristics Correlate With Multiple Factors That Predict Central Lymph Node Metastasis of Papillary Thyroid Carcinoma: Significant Role of HBME-1. Eur J Radiol (2020) 123:108801. doi: 10.1016/j.ejrad.2019.108801

24. Shaha AR, Shah JP, Loree TR. Differentiated Thyroid Cancer Presenting Initially With Distant Metastasis. Am J Surg (1997) 174(5):474-6. doi: 10.1016/s0002-9610(97)00158-x 
25. Back K, Kim JS, Kim JH, Choe JH. Superior Located Papillary Thyroid Microcarcinoma is a Risk Factor for Lateral Lymph Node Metastasis. Ann Surg Oncol (2019) 26(12):3992-4001. doi: 10.1245/s10434-019-07587-2

26. Fiore E, Rago T, Latrofa F, Provenzale MA, Piaggi P, Delitala A, et al. Hashimoto's Thyroiditis is Associated With Papillary Thyroid Carcinoma: Role of TSH and of Treatment With L-Thyroxine. Endocr Relat Cancer (2011) 18(4):429-37. doi: 10.1530/ERC-11-0028

27. Kim SS, Lee BJ, Lee JC, Song SH, Kim BH, Son SM, et al. Preoperative Serum Thyroid Stimulating Hormone Levels in Well-Differentiated Thyroid Carcinoma is a Predictive Factor for Lateral Lymph Node Metastasis as Well as Extrathyroidal Extension in Korean Patients: A Single-Center Experience. Endocrine (2011) 39(3):259-65. doi: 10.1007/s12020-010-9430-5

28. Kohler H, Latteyer S, Hones GS, Theurer S, Liao XH, Christoph S, et al. Increased Anaplastic Lymphoma Kinase Activity Induces a Poorly Differentiated Thyroid Carcinoma in Mice. Thyroid (2019) 29(10):1438-46. doi: $10.1089 /$ thy.2018.0526

29. Fiore E, Rago T, Provenzale MA, Scutari M, Ugolini C, Basolo F, et al. Lower Levels of TSH are Associated With a Lower Risk of Papillary Thyroid Cancer in Patients With Thyroid Nodular Disease: Thyroid Autonomy may Play a Protective Role. Endocr Relat Cancer (2009) 16(4):1251-60. doi: 10.1677/ERC-09-0036

30. Ito $\mathrm{Y}$, Miyauchi A, Inoue H, Fukushima M, Kihara M, Higashiyama $\mathrm{T}$, et al. An Observational Trial for Papillary Thyroid Microcarcinoma in Japanese Patients. World J Surg (2010) 34(1):28-35. doi: 10.1007/s00268-009-0303-0

31. Yu XM, Wan Y, Sippel RS, Chen H. Should All Papillary Thyroid Microcarcinomas be Aggressively Treated? An Analysis of 18,445 Cases. Ann Surg (2011) 254(4):653-60. doi: 10.1097/SLA.0b013e318230036d

32. Maciel J, Donato S, Simões H, Leite V. Clinical Outcomes of a Conservative Approach in Cervical Lymph Node Metastases of Thyroid Cancer. Clin Endocrinol (Oxf) (2020) 94(3):460-5. doi: 10.1111/cen.14306

33. Qubain SW, Nakano S, Baba M, Takao S, Aikou T. Distribution of Lymph Node Micrometastasis in Pn0 Well-Differentiated Thyroid Carcinoma. Surgery (2002) 131(3):249-56. doi: 10.1111/cen.14306

34. Likhterov I, Reis LL, Urken ML. Central Compartment Management in Patients With Papillary Thyroid Cancer Presenting With Metastatic Disease to the Lateral Neck: Anatomic Pathways of Lymphatic Spread. Head Neck (2017) 39(5):853-9. doi: 10.1002/hed.24568

35. Hu D, Zhou J, He W, Peng J, Cao Y, Ren H, et al. Risk Factors of Lateral Lymph Node Metastasis in Cn0 Papillary Thyroid Carcinoma. World J Surg Oncol (2018) 16(1):30. doi: 10.1186/s12957-018-1336-3

36. Zhang TT, Qi XZ, Chen JP, Shi RL, Wen SS, Wang YL, et al. The Association Between Tumor's Location and Cervical Lymph Nodes Metastasis in Papillary Thyroid Cancer. Gland Surg (2019) 8(5):557-68. doi: 10.21037/gs.2019.10.02

37. Bansal M, Gandhi M, Ferris RL, Nikiforova MN, Yip L, Carty SE, et al. Molecular and Histopathologic Characteristics of Multifocal Papillary Thyroid Carcinoma. Am J Surg Pathol (2013) 37(10):1586-91. doi: 10.1097/ PAS.0b013e318292b780

38. Wang W, Su X, He K, Wang Y, Wang H, Wang H, et al. Comparison of the Clinicopathologic Features and Prognosis of Bilateral Versus Unilateral Multifocal Papillary Thyroid Cancer: An Updated Study With More Than 2000 Consecutive Patients. Cancer (2016) 122(2):198-206. doi: 10.1002/ cncr.29689

39. Dong S, Xie XJ, Xia Q, Wu YJ. Indicators of Multifocality in Papillary Thyroid Carcinoma Concurrent With Hashimoto's Thyroiditis. Am J Cancer Res (2019) 9(8):1786-95.

40. Dou Y, Chen Y, Hu D, Su X. The Recovery of Thyroid Function in Low-Risk Papillary Thyroid Cancer After Lobectomy: A 3-Year Follow-Up Study. Front Endocrinol (Lausanne) (2020) 11:619841. doi: 10.3389/fendo.2020.619841
41. Kim SK, Park I, Woo JW, Lee JH, Choe JH, Kim JH, et al. Predictive Factors for Lymph Node Metastasis in Papillary Thyroid Microcarcinoma. Ann Surg Oncol (2016) 23(9):2866-73. doi: 10.1245/s10434-016-5225-0

42. Kitahara CM, Pfeiffer RM, Sosa JA, Shiels MS. Overweight and Obesity on US Papillary Thyroid Cancer Incidence Trends (1995-2015). J Natl Cancer Inst (2020) 112(8):810-7. doi: 10.1093/jnci/djz202

43. Kwon H, Han KD, Park CY. Weight Change is Significantly Associated With Risk of Thyroid Cancer: A Nationwide Population-Based Cohort Study. Sci Rep (2019) 9(1):1546. doi: 10.1038/s41598-018-38203-0

44. Kang YE, Kim JT, Lim MA, Oh C, Liu L, Jung SN, et al. Association Between Circulating Fibroblast Growth Factor 21 and Aggressiveness in Thyroid Cancer. Cancers (Basel) (2019) 11(8). doi: 10.3390/cancers11081154

45. Ray A, Cleary MP. The Potential Role of Leptin in Tumor Invasion and Metastasis. Cytokine Growth Factor Rev (2017) 38:80-97. doi: 10.1016/ j.cytogfr.2017.11.002

46. Grani G, Lamartina L, Montesano T, Ronga G, Maggisano V, Falcone R, et al. Lack of Association Between Obesity and Aggressiveness of Differentiated Thyroid Cancer. J Endocrinol Invest. (2019) 42(1):85-90. doi: 10.1007/s40618018-0889-x

47. Paes JE, Hua K, Nagy R, Kloos RT, Jarjoura D, Ringel MD. The Relationship Between Body Mass Index and Thyroid Cancer Pathology Features and Outcomes: A Clinicopathological Cohort Study. J Clin Endocrinol Metab (2010) 95(9):4244-50. doi: 10.1210/jc.2010-0440

48. Jara SM, Carson KA, Pai SI, Agrawal N, Richmon JD, Prescott JD, et al. The Relationship Between Chronic Lymphocytic Thyroiditis and Central Neck Lymph Node Metastasis in North American Patients With Papillary Thyroid Carcinoma. Surgery (2013) 154(6):1272-80. doi: 10.1016/j.surg.2013.07.021 discussion 1280-1282.

49. Wen X, Wang B, Jin Q, Zhang W, Qiu M. Thyroid Antibody Status is Associated With Central Lymph Node Metastases in Papillary Thyroid Carcinoma Patients With Hashimoto's Thyroiditis. Ann Surg Oncol (2019) 26(6):1751-8. doi: 10.1245/s10434-019-07256-4

50. Thompson AM, Turner RM, Hayen A, Aniss A, Jalaty S, Learoyd DL, et al. A Preoperative Nomogram for the Prediction of Ipsilateral Central Compartment Lymph Node Metastases in Papillary Thyroid Cancer. Thyroid (2014) 24(4):675-82. doi: 10.1089/thy.2013.0224

51. Wang Y, Guan Q, Xiang J. Nomogram for Predicting Central Lymph Node Metastasis in Papillary Thyroid Microcarcinoma: A Retrospective Cohort Study of 8668 Patients. Int J Surg (2018) 55:98-102. doi: 10.1016/j.ijsu.2018.05.023

Conflict of Interest: The authors declare that the research was conducted in the absence of any commercial or financial relationships that could be construed as a potential conflict of interest.

Publisher's Note: All claims expressed in this article are solely those of the authors and do not necessarily represent those of their affiliated organizations, or those of the publisher, the editors and the reviewers. Any product that may be evaluated in this article, or claim that may be made by its manufacturer, is not guaranteed or endorsed by the publisher.

Copyright (อ 2021 Shu, Tang, Hu, Wang, Yu, Yang, Deng, Wang and Su. This is an open-access article distributed under the terms of the Creative Commons Attribution License (CC BY). The use, distribution or reproduction in other forums is permitted, provided the original author(s) and the copyright owner(s) are credited and that the original publication in this journal is cited, in accordance with accepted academic practice. No use, distribution or reproduction is permitted which does not comply with these terms. 